\title{
Emittance and energy spread compensation for current and future low emittance synchrotron light sources
}

\author{
D. Hidas, ${ }^{*}$ T. Shaftan, and T. Tanabe \\ Brookhaven National Laboratory, Upton, New York 11973, USA
}

(National Synchrotron Light Source II)

(Received 26 February 2021; accepted 26 July 2021; published 20 August 2021)

\begin{abstract}
This paper presents the results of an analysis focused on the behavior of emittance, energy spread and radiated power, as well as their variations in relation to the complement of active insertion devices in National Synchrotron Light Source II (NSLS-II) operations. The spectra of radiated power of the installed insertion devices and analytic values of the emittance are compared to that measured by the NSLS-II online diagnostics. A conceptual design of a compensation wiggler that will maintain a constant emittance or energy spread is investigated for NSLS-II and a potential low emittance upgrade scenario.
\end{abstract}

DOI: 10.1103/PhysRevAccelBeams.24.081601

\section{INTRODUCTION}

The National Synchrotron Light Source II (NSLS-II) storage ring is a $3 \mathrm{GeV}$ electron storage ring with the potential to house upwards of 60 photon extraction beam lines. Currently the typical operating horizontal emittance $\left(\epsilon_{x}\right)$ is around $0.78 \mathrm{~nm}$ rad with an electron beam energy spread $\left(\sigma_{E} / E\right)$ of $0.0826 \%$. Excluding damping wigglers (DW) which have constant field during operations there are 17 insertion devices (ID) [1] whose field varies according to the immediate demands of their respective individual beam-line users. These IDs affect the total radiated power from synchrotron radiation and in turn contributions to the synchrotron radiation integrals. The effect on the horizontal emittance due to these changes is both calculable and measurable and is the subject of this paper.

Beam-line techniques such as scanning transmission $\mathrm{x}$-ray microscopy and microfocus beam lines which are very sensitive to variations of the transverse photon distribution can be significantly affected by changes in the horizontal emittance, particularly at the very low emittances currently being realized. Several methods have previously been proposed to compensate insertion device induced emittance variations [2]. Bunch lengthening by means of a harmonic cavity to alter the intrabeam scattering properties to stabilize the emittance may be possible within

\footnotetext{
*dhidas@bnl.gov

Published by the American Physical Society under the terms of the Creative Commons Attribution 4.0 International license. Further distribution of this work must maintain attribution to the author(s) and the published article's title, journal citation, and DOI.
}

constraints imposed by lifetime and beam induced heating requirements. Adding a variable dispersion bump in a fixed-gap wiggler [3] for compensation may be possible if space affords for the required non-negligible dispersion bump and effects on beam dynamics understood. This paper explores the viability of a variable-gap wiggler compensation in a dedicated dispersion free straight section having the benefits of operating at a lower emittance and assurance of electron beam stability.

Both the current NSLS-II and an example upgrade lattice (designated herein simply as UL) with significantly lower emittance are investigated using the same online insertion device data gathered from a normal operations period of NSLS-II over 24 hours on October 25, 2019. These data are used to show the variation of emittance and energy spread for NSLS-II and what one may reasonably expect from a similar $3 \mathrm{GeV}$ low emittance lattice.

A hypothetical compensation wiggler (CW) is then modeled with the same period for NSLS-II and example upgrade lattice which is capable of providing a compensated constant horizontal emittance or constant energy spread.

\section{NSLS-II EMITTANCE AND SPECTRAL POWER}

The NSLS-II electron storage ring consists of 30 doublebend achromat cells (having near-zero dispersion in the ID straight sections) and is well described elsewhere [4]. The accelerator parameters of interest in this study are the synchrotron radiation integrals $I_{2}$ through $I_{5}$ (found in many accelerator texts, for example [5]) reproduced in Eq. (1), horizontal emittance, electron beam energy spread, and horizontal beta functions in the long $\left(\beta_{x}^{l s}\right)$ and short 
TABLE I. Bare lattice synchrotron radiation integrals, energy spread, horizontal emittance, beam energy, and beta functions at the lattice waist of the short straight (ss) and long straight $(l s)$ sections.

\begin{tabular}{lccc}
\hline \hline & NSLS-II & Upgrade lattice & Units \\
\hline$I_{2 b}$ & $2.51 \times 10^{-1}$ & $2.17 \times 10^{-1}$ & $1 / \mathrm{m}$ \\
$I_{3 b}$ & $1.00 \times 10^{-2}$ & $8.35 \times 10^{-3}$ & $1 / \mathrm{m}^{2}$ \\
$I_{4 b}$ & $-2.30 \times 10^{-4}$ & $-2.15 \times 10^{-1}$ & $1 / \mathrm{m}$ \\
$I_{5 b}$ & $3.97 \times 10^{-5}$ & $8.31 \times 10^{-7}$ & $1 / \mathrm{m}$ \\
$\sigma_{E} / E$ & $5.14 \times 10^{-4}$ & $7.09 \times 10^{-4}$ & \\
$\epsilon_{x b}$ & 2.09 & 0.025 & $\mathrm{~nm} \mathrm{rad}$ \\
$E$ & 3 & 3 & $\mathrm{GeV}$ \\
$\beta_{x}^{s s}$ & 1.8 & 1.9 & $\mathrm{~m}$ \\
$\beta_{x}^{l s}$ & 20.5 & 20.2 & $\mathrm{~m}$ \\
\hline \hline
\end{tabular}

$\left(\beta_{x}^{s s}\right)$ straight sections of the current NSLS-II storage ring and example candidate upgrade lattice:

$I_{2}=\oint \frac{1}{\rho^{2}} d s$

$I_{3}=\oint \frac{1}{|\rho|^{3}} d s$

$I_{4}=\oint \frac{\eta}{\rho}\left(\frac{1}{\rho^{2}}+2 k_{1}\right) d s, \quad k_{1}=\frac{e}{P_{0}} \frac{\partial B_{y}}{\partial x}$

$I_{5}=\oint \frac{\mathcal{H}_{x}}{|\rho|^{3}} d s, \quad \mathcal{H}_{x}=\gamma_{x} \eta_{x}^{2}+2 \alpha_{x} \eta_{x} \eta_{p x}+\beta_{x} \eta_{p x}^{2}$.

For the current NSLS-II storage ring the parameters of interest are computed from the standard NSLS-II bare (without damping wigglers) lattice ELEGANT [6] model and are given in Table I along with the parameters from the similarly computed example candidate low emittance upgrade design based on a complex bend model following [7]. The NSLS-II bare lattice horizontal emittance calculated from this model is $2.086 \mathrm{~nm}$ rad which is reduced to $0.917 \mathrm{~nm}$ rad with the inclusion of three sets of damping wigglers, with a rise in the electron beam energy spread from $0.051 \%$ to $0.084 \%$.

The remainder of the suite of NSLS-II insertion devices given in Table II reduces slightly both the energy spread and horizontal emittance when operated at their maximum effective deflection parameter $\left(K_{\text {eff }}\right)$, or equivalently highest effective magnetic field $\left(B_{\text {eff }}\right)$. All devices operating at their respective maximum deflection parameter gaps correspond to a calculated $\epsilon_{x b}=0.727 \mathrm{~nm} \mathrm{rad}$ and $\epsilon_{x b}=0.0822 \%$. The corresponding total spectral power at maximum $K_{\text {eff }}$ is shown in Fig. 1 along with the bending magnet (BM) and damping wiggler spectra for comparison. The critical energies for the IDs and ring sum are 5087 and $4766 \mathrm{eV}$ corresponding to an equivalent bending magnet $\rho$ of 11.8 and $12.6 \mathrm{~m}$ respectively, as compared to the bending magnets themselves at $25 \mathrm{~m}$. The equivalent bending magnet $\rho$ is expected to be further reduced with the near
TABLE II. Insertion devices at NSLS-II.

\begin{tabular}{lccr}
\hline \hline Type/Name & $\lambda_{W}(\mathrm{~mm})$ & $L_{W}(\mathrm{~m})$ & $K_{\text {eff }}^{\max }$ \\
\hline DW100 & 100 & 6.8 & 17.2 \\
DW100 & 100 & 6.8 & 17.2 \\
DW100 & 100 & 6.8 & 17.2 \\
IVU18 & 18 & 1.0 & 1.5 \\
IVU20 & 20 & 3.0 & 1.8 \\
IVU20 & 20 & 3.0 & 1.8 \\
IVU21 & 21 & 1.5 & 1.7 \\
IVU21 & 21 & 1.5 & 1.7 \\
IVU21 & 21 & 1.5 & 1.7 \\
IVU22 & 22 & 3.0 & 1.5 \\
IVU23 & 23 & 2.8 & 2.0 \\
IVU23 & 23 & 2.8 & 2.0 \\
IVU23 & 23 & 2.8 & 2.0 \\
U42 & 42 & 1.4 & 3.7 \\
EPU49 & 49 & 2.0 & 4.3 \\
EPU49 & 49 & 2.0 & 4.3 \\
EPU57 & 57 & 3.5 & 4.4 \\
EPU57 & 57 & 1.4 & 4.4 \\
EPU60 & 60 & 0.9 & 5.7 \\
EPU105 & 105 & 2.7 & 11.2 \\
\hline \hline
\end{tabular}

future addition of a high field superconducting wiggler, an additional in-vacuum undulator (IVU), and elliptically polarizing undulators (EPU) for the NEXT-II project, as well as future devices.

The magnetic gaps of all but the damping wiggler insertion devices of Table II are typically frequently changing to satisfy the requirements of many different and simultaneous experiments requiring vastly different photon beam properties. Consequently, the spectral power output for IDs (ID curve of Fig. 1) and hence the horizontal emittance are constantly varying quantities.

Both the current NSLS-II lattice and a future low emittance model are investigated. The particularities of the upgrade lattice shown here are less consequential than

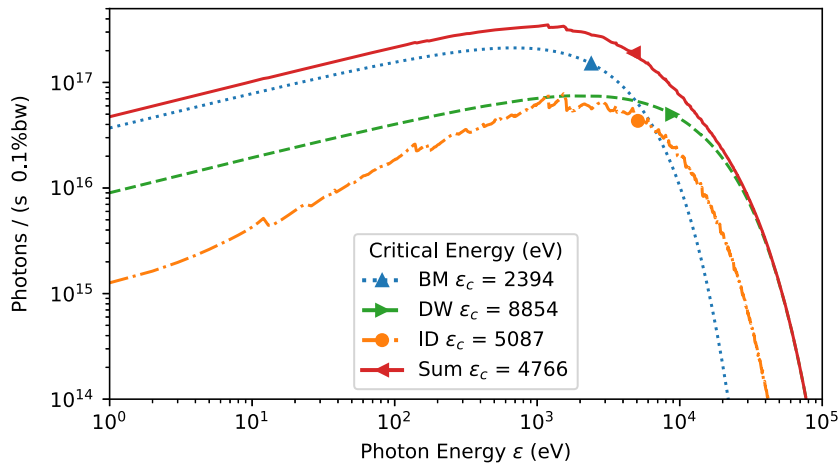

FIG. 1. Total spectral power by source type for NSLS-II at $400 \mathrm{~mA}$ calculated using SPECTRA [8]. The ID curve is a summation of all nondamping wiggler IDs at their maximum $K_{\text {eff }}$ (minimum magnetic gap). The critical energy $\epsilon_{c}$ is taken such that half of the integral is both above and below. 
the overall paradigm shift to a significantly lower emittance design. All low emittance upgrade lattices tested behave similarly and the choice of this one in particular bears no particular significance.

The effects of insertion devices on the horizontal emittance and energy spread in storage rings are well described by their contributing synchrotron radiation integrals. The relevant wiggler integrals are given in Eq. (2). Here $L_{W}$ is the length of the device, $\rho_{W}$ the minimum radius of curvature associated with the maximum vertical magnetic field, $\lambda_{W}$ the period, and $\left\langle\beta_{x}\right\rangle_{W}$ the average of the horizontal betatron function over the length of the device:

$$
\begin{aligned}
I_{2 W} & =\frac{L_{W}}{2 \rho_{W}^{2}} \\
I_{3 W} & =\frac{4 L_{W}}{3 \pi \rho_{W}^{3}} \\
I_{4 W} & =0 \\
I_{5 W} & =\frac{\lambda_{W}^{2}\left\langle\beta_{x}\right\rangle_{W} L_{W}}{15 \pi^{3} \rho_{W}^{5}} .
\end{aligned}
$$

The effect of a suite of insertion devices on the horizontal emittance $\epsilon_{x}$ and energy spread $\sigma_{E}$ can be expressed through their radiation integrals as shown in Eqs. (3) and (4), where $\epsilon_{x b}$ and $\sigma_{E b}$ denote the horizontal emittance and energy spread respectively of the bare lattice. Carefully calibrated measurements of the horizontal emittance and energy spread for NSLS-II have been shown to be in very good agreement with this formalism [9].

$$
\begin{gathered}
\epsilon_{x}=\epsilon_{x b} \frac{1+\frac{\sum_{I D s} I_{5 W}^{I D}}{I_{5 b}}}{1+\frac{\sum_{I D s}\left(I_{2 W}^{I D}-I_{4 W}^{I D}\right)}{I_{2 b}-I_{4 b}}} \\
\sigma_{E}=\sigma_{E b} \sqrt{\frac{1+\frac{\sum_{I D s} I_{3 W}^{I D}}{I_{3 b}}}{1+\frac{\sum_{I D s}\left(2 I_{2 W}^{I D}+I_{4 W}^{I D}\right)}{2 I_{2 b}+I_{4 b}}}} .
\end{gathered}
$$

The full list of IDs considered in this analysis includes three sets of damping wigglers (DW), ten IVUs, six EPUs, and one out of vacuum undulator (U). The relevant parameters for these IDs are given in Table II. The evolution of the horizontal emittance and energy spread with the addition of each insertion device installed at NSLS-II at its maximum $K_{\text {eff }}$ (minimum magnetic gap) is shown in Fig. 2. The bare lattice is shown on the left followed by the damping wigglers (DW) and the remaining insertion devices in order of increasing magnetic period. This plot represents the cumulative effect of closing each device starting on the left with the bare lattice and moving towards the right and illustrates the potential effect each has on the emittance and energy spread. The actual ID configuration in operations varies continuously and is the subject of Sec. III.

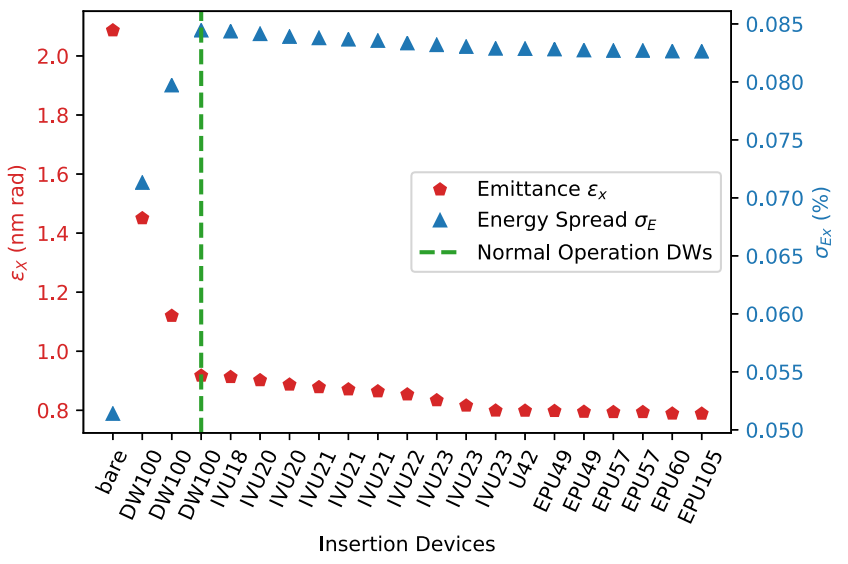

FIG. 2. Calculated emittance and energy spread starting on the left from the NSLS-II bare lattice of Table I cumulatively adding each of the installed IDs at their respective maximum effective deflection parameter $\left(K_{\text {eff }}\right)$ of Table II.

\section{NSLS-II INSERTION DEVICE OPERATION}

The magnetic gap of each ID installed at NSLS-II is monitored and archived with a minimum frequency of $1 \mathrm{~Hz}$ and is for purposes of this analysis down-sampled to $1 \mathrm{~Hz}$. The nominal operating beam current during this period was $400 \mathrm{~mA}$. The data is selected such that the beam current is always above $398 \mathrm{~mA}$, the online "operations" flag must be set (indicating normal user operations conditions), and all IDs are required to be operating in linear horizontal (with respect to photon polarization) mode where the horizontal magnetic field is negligibly close to zero. The portions in this time window noticeably removed from this analysis do not satisfy at least one of these conditions.

\section{A. Insertion device magnetic field}

In order to understand the evolution of the effect on emittance and energy spread from the IDs during operations it is necessary to know the device properties and the magnetic field at each point in time.

The magnetic field of every insertion device installed in the NSLS-II storage ring is characterized in detail before installation [10]. The measurements used herein are field mappings taken along the longitudinal centerline of each device at different magnetic gaps using a 3D hall probe with a measurement pitch of $647.6 \mu \mathrm{m}$ longitudinally. From these data the peak effective magnetic field $B_{\text {eff }}$, excluding the end termination regions, is calculated for each gap measured for each device. For each device the period is determined numerically from peak finding and subsequently a discrete Fourier transform gives the magnetic field harmonic components $B_{n}$ from which one can compute $B_{\text {eff }}=\sqrt{\sum_{n}^{\infty} B_{n}^{2} / n^{2}}$ choosing a practical cutoff for the summation. A piecewise natural logarithmic spline is then used as the interpolating function from gap to effective magnetic field. This ensures monotonic 


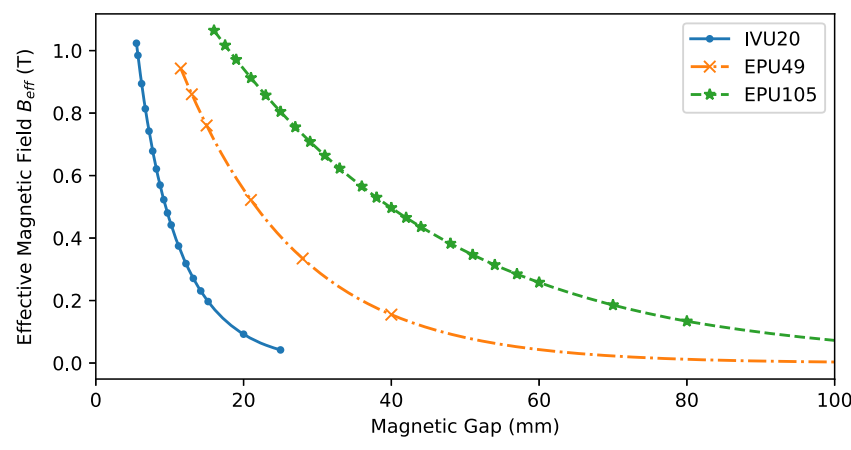

FIG. 3. Insertion device magnetic field as a function of gap shown for an IVU with a $20(\mathrm{~mm})$ period and two EPUs of periods 49 and $105(\mathrm{~mm})$. The lines indicate the interpolated curve while the points indicate the magnetic gap at which the field was measured.

decreasing behavior with occasional sparse data where the typical cubic spline may have poor behavior in the sparse tails. Figure 3 shows an example of these data and interpolating functions for three IDs of different periods.

Once gap to magnetic field interpolating functions is created the effective magnetic field for each ID during operations can be easily calculated at any gap. The $1 \mathrm{~Hz}$ ID magnetic gap data is run through interpolating functions similar to Fig. 3 to give the effective magnetic field during operations each second for every ID at NSLS-II. These data will later be used to directly calculate the $\rho_{W}$ (bending radius) of Eq. (2) when assessing the synchrotron radiation integrals at each sampled time.

\section{B. Insertion device power in operations}

With the on-axis longitudinal magnetic field mapping for every ID it is possible to calculate the electron beam trajectory for every magnetic gap measured for each device. This trajectory is corrected using simulated magnetic kicks on the end of each ID to remove remnant first and second field integral components such that the exit trajectory has zero transverse displacement and momentum. The trajectory itself is calculated using a standard fourth-order Runge-Kutta method with initial conditions representing the $3(\mathrm{GeV})$ electron beam of NSLS-II at the entrance of each ID, which is then propagated forward in time. The magnetic field measurement pitch of 1544 points per meter is not sufficient for accurate trajectory propagation. A third-order spline is used for interpolation of the magnetic field to achieve an accurate trajectory propagation.

Once a trajectory is established for each magnetic gap the total power at each gap can be calculated using the well known formula given in Eq. (5). Here $e$ is the electron charge, $\gamma$ the relativistic factor $1 / \sqrt{1-|\vec{v}|^{2} / c^{2}}$, I the beam current, $\epsilon_{0}$ the permittivity of free space, $c$ the speed of light in vacuum, and $\vec{a}$ and $\vec{v}$ the acceleration and velocity

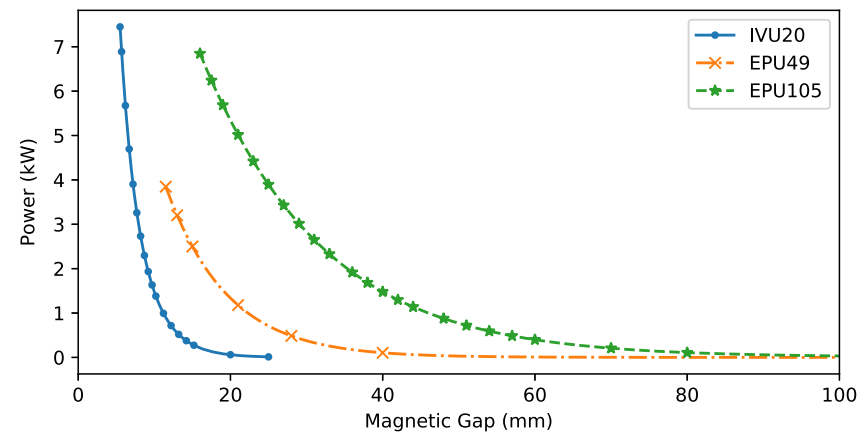

FIG. 4. Insertion device power at a beam current of $400(\mathrm{~mA})$ as a function of gap shown for an IVU with a $20(\mathrm{~mm})$ period and two EPUs of periods 49 and $105(\mathrm{~mm})$. The lines indicate the interpolated curve while the points indicate the magnetic gap at which the field was measured.

respectively. These are the methods implemented in the OSCARS software [11]:

$$
P_{\text {total }}=\frac{e^{2} \gamma^{6}}{6 \pi \epsilon_{0} c} \frac{I}{e} \int_{-\infty}^{\infty}\left[\vec{a}^{2}-(\vec{v} \times \vec{a})^{2}\right] \mathrm{d} t
$$

In the calculation of total power a filament beam is always assumed. The small emittance and hence small transverse momentum spread in the insertion device straight sections make this a highly accurate and valid assumption at nearly all current synchrotron light source facilities. The limits of integration are truncated outside the field of the device where $|\vec{a}|$ is obviously zero. The integration is done iteratively such that in the $n$th iteration there are $2^{n+1}-1$ points total. The numerical integral for $P_{\text {total }}^{n}$ is compared with $P_{\text {total }}^{n-1}$ until the desired relative precision is achieved, which in this case is $0.1 \%$. Where intermediary trajectory points are needed they are derived from a cubic spline.

Figure 4 shows an example of the results of these total power calculations for three IDs as a function of magnetic gap at the nominal operating current of $400(\mathrm{~mA})$ for NSLS-II where the points indicate where magnetic field measurements were taken. The lines are again a piecewise natural logarithmic interpolation similar to Fig. 3, which are used to calculate the total power at any gap, which will be weighted by the online beam current each second.

Using the magnetic field data for each device described in Sec. III and interpolating curves similar to Fig. 4, the total power output can be calculated for each device as a function of time according to the instantaneous beam current. The result of this calculation is shown in Fig. 5 for three IDs as well as the sum of all IDs excluding the damping wigglers. A longer duration of 1 month with a sampling period of 1 minute from November 2020 similarly showed that the ID power output on average was $42.3 \mathrm{~kW}$ with a standard deviation of $1.7 \mathrm{~kW}$. A detailed approach is taken here for the calculation of ID power, 


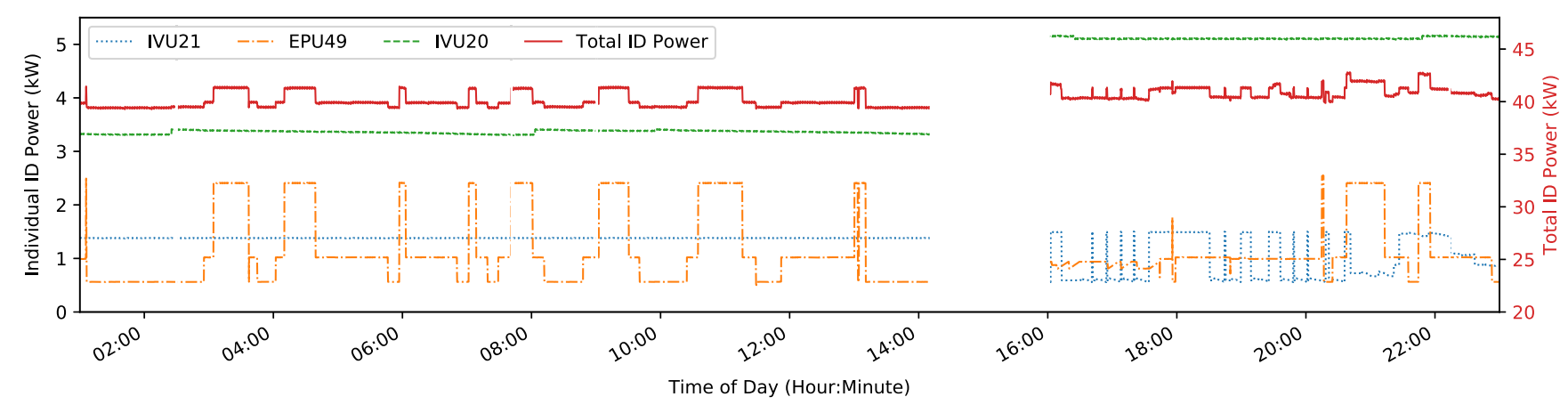

FIG. 5. Undulator power for selected IDs and the summed total for all IDs excluding damping wigglers during operations calculated using the online magnetic gap read-back value and measured magnetic field data for each ID.

but it is noted that for some purposes the analytic expression of Eq. (6), where $Z_{0}$ is the vacuum impedance, may suffice and is the method used to calculate the power for the hypothetical compensating wigglers in subsequent sections:

$$
P=\frac{L_{W}}{6} Z_{0} I e \frac{2 \pi c}{\lambda_{W}^{2}} \gamma^{2} K^{2}
$$

It is interesting to compare the total power output of these insertion devices to the online emittance measurements as seen in Fig. 6 where the correlation between total power and horizontal emittance can be clearly seen. It should be noted that the NSLS-II ring has a large damping wiggler power contribution relative to the beam line insertion devices and other rings not dominated in this way may see an even more pronounced effect. The online emittance is measured using a pinhole camera [12] where slow drift variations are typically of order $\pm 5 \%$. The emittance data shown herein is shifted downward by $31 \mathrm{pm}$ rad to correct for this drift for ease of comparison to calculated emittance values as the fluctuations of interest are small and arising from ID movement.

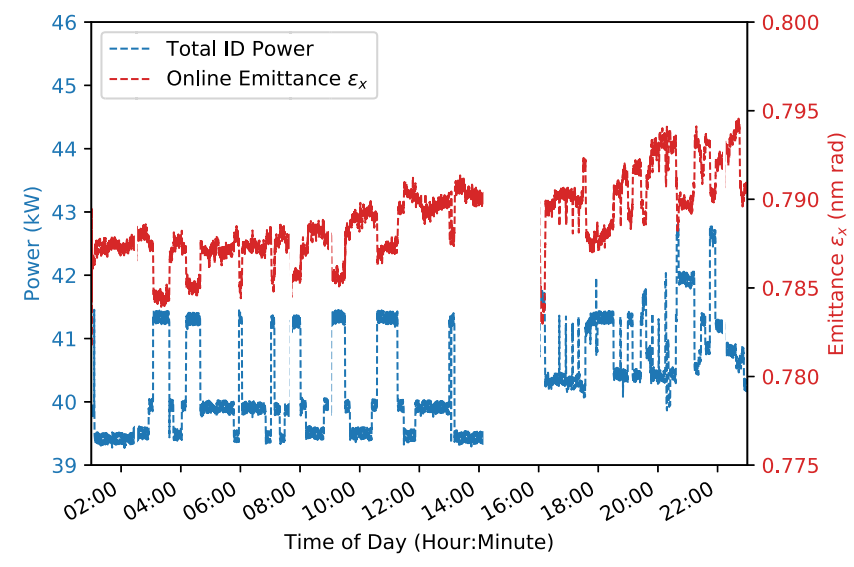

FIG. 6. Total power for all IDs (damping wigglers excluded) shown with the online emittance measurement for the 24-hour period used throughout.

\section{ID FIELD-BASED CALCULATION OF EMITTANCE}

Returning to Eqs. (3) and (4) it is now possible to calculate the evolution of the horizontal emittance and energy spread in time given the magnetic field data for every device in Sec. III and the wiggler integrals in Eq. (2) where $\rho_{W}$ is related to the magnetic field such that $\rho_{W}=\frac{\beta E}{B e}$. The results of this calculation are given in Fig. 7 and compared to the online emittance measurement. It can be seen very clearly in this figure that the emittance calculated from online ID gap values describes very well the behavior observed in the independent emittance measurement. The emittance measurement in Figs. 7 and 6 is shown as a running average over a 1 minute interval to reduce statistical error.

\section{EMITTANCE AND ENERGY SPREAD COMPENSATION}

Looking at Eq. (3) it is possible to envision a device with appropriate behavior of the synchrotron integrals in Eq. (2) which will compensate for the changing fields of all other

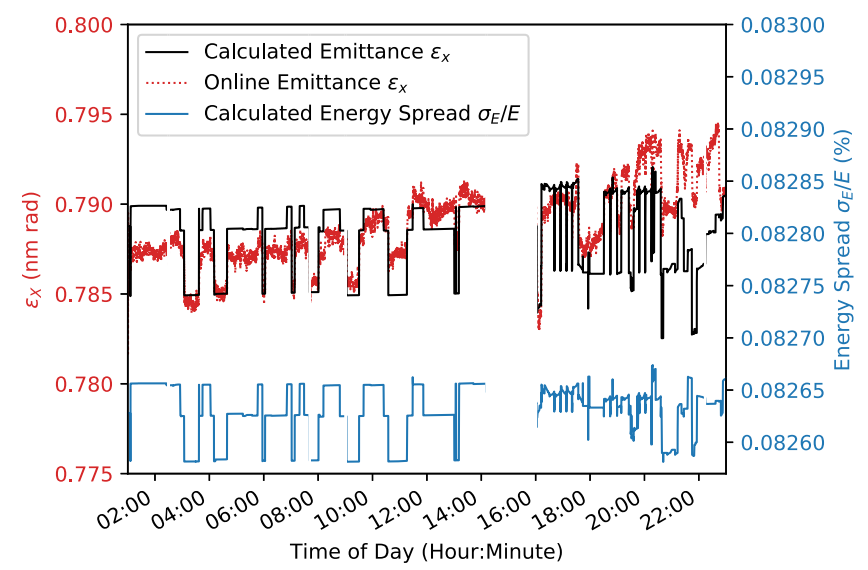

FIG. 7. Calculated emittance and energy spread based on insertion device online gap measurements and interpolated magnetic fields from lab measurements. 

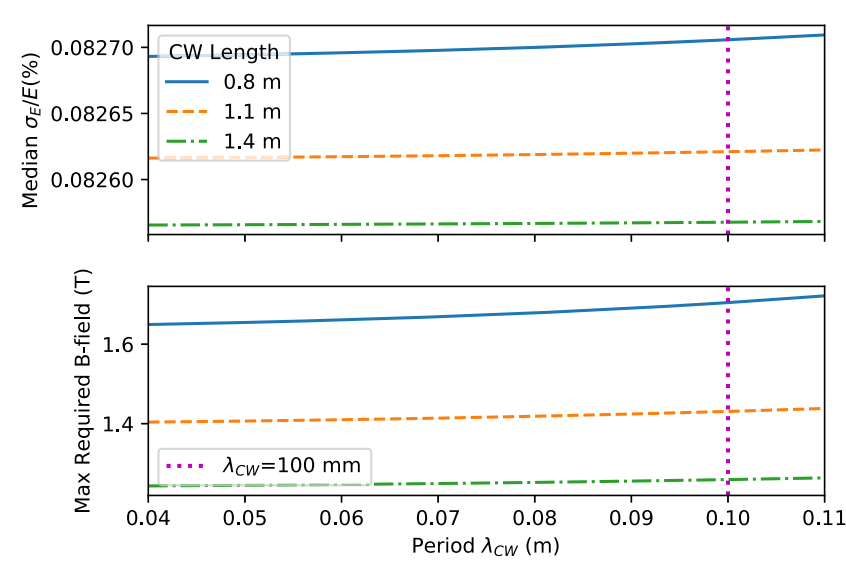

FIG. 8. Median energy spread $\sigma_{E}$ (top) and maximum required magnetic field required for compensation wigglers (bottom) of different periods and lengths required to maintain the specified horizontal emittance $\epsilon_{x}^{c}=0.780 \mathrm{~nm} \mathrm{rad}$.

devices included in the sum $\sum_{I D}$ which is capable of keeping either the horizontal emittance or energy spread constant, or some other desirable minimization of the combination of these. To see this clearly one device labeled $\mathrm{CW}$ is separated from the summation and written explicitly in Eqs. (7) and (8) substituting the CW contributions from Eq. (2). The purpose of this will be to identify the appropriate parameters for a compensating wiggler such that variation of the magnetic field (magnetic gap) will suffice in accomplishing the compensation and that such a device is practically achievable. In this respect length, minimum gap, period, and magnetic strength should all be considered:

$$
\begin{gathered}
\epsilon_{x}^{c}=\epsilon_{b} \frac{1+\frac{1}{I_{5 b}} \sum_{I D s} I_{5 W}^{I D}+\frac{\left\langle\beta_{x}\right\rangle_{C W} B_{C W}^{5} e^{5} L_{C W} \lambda_{C W}^{2}}{15 c^{5} m^{3} \pi^{3} \beta^{5} \gamma^{5} I_{5 b}}}{1+\left(\frac{\sum_{I D s}\left(I_{2 W}^{I D}-I_{4 W}^{I D}\right)}{I_{2 b}-I_{4 b}}\right)+\frac{B_{C W}^{2} e^{2} L_{C W}}{2 c^{2} m^{2} \beta^{2} \gamma^{2}\left(I_{2 b}-I_{4 b}\right)}} \\
\sigma_{E}^{c}=\sigma_{b} \sqrt{\frac{1+\left(1+\frac{\sum_{I D s} I_{3 W}^{I D}}{I_{3 b}}\right)+\frac{4 B_{C W}^{3} e^{3} L_{C W}}{3 c^{3} m^{3} \pi \beta^{3} \gamma^{3} I_{3 b}}}{1+\frac{B_{C W}^{2} e^{2} L_{C W}}{c^{2} m^{2} \beta^{2} \gamma^{2}\left(2 I_{2 b}+I_{4 b}\right)}}}
\end{gathered}
$$

If in Eq. (7) one assumes $\epsilon_{x}^{c}$ constant this leaves an equation of three unknowns in which there may exist at least one value of $B_{C W}$ for a given length $L_{C W}$ and period $\lambda_{C W}$ that is capable of maintaining the specified value of $\epsilon_{x}^{c}$. The choice of $L_{C W}$ and $\lambda_{C W}$ is crucial in designing a compensation wiggler in that $B_{C W}$ must be physically achievable and that this choice may dramatically affect the energy spread governed by Eq. (8). It is noted here that $\left\langle\beta_{x}\right\rangle_{C W}$ is determined by $L_{C W}$ and the placement in the lattice, which here is taken to be centered at the waist (where the Twiss parameter $\alpha=0$ ) of the long straight section so that $\beta_{x}=\beta_{x}^{l s}$.

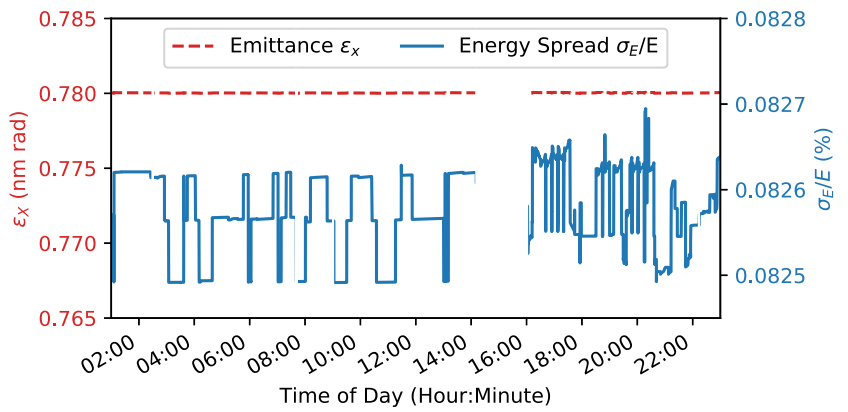

FIG. 9. Calculated emittance and energy spread with CW100 providing constant emittance $\epsilon_{x}^{c}=0.780 \mathrm{~nm} \mathrm{rad}$.

The relationship of $L_{C W}, \lambda_{C W}$, and $B_{C W}$ is somewhat complicated and warrants concrete exemplification. As an example one can reasonably choose $\epsilon_{x}^{c}=0.780 \mathrm{~nm} \mathrm{rad}$, which is slightly below the minimum calculated horizontal emittance for the data in Fig. 7. One is then free to choose $L_{C W}$ and $\lambda_{C W}$ which will then determine the values $B_{C W}$ as a function of time necessary to maintain the desired emittance state. Three such length choices for this are shown in Fig. 8 where the maximum $B_{C W}$ is shown as a function of $\lambda_{C W}$. It is important to remember here for a given choice of length and period that $B_{C W}$ must change to do the desired compensation and it is the maximum value of that time series data which is shown. The upper plot in Fig. 8 is similarly the median energy spread for the same configuration of length and period.

This illustration suggests two conclusions. One is that the shorter the compensation device is the more adverse effect it will have on the energy spread due to the larger field required. The second is that a device with a shorter period will require a lower maximum magnetic field. In practice this will be subject to some practical limitations of accelerator real estate and magnet technology that one should be keenly aware of in designing such a device.

As an example of emittance compensation for NSLS-II take $\lambda_{C W}=100 \mathrm{~mm}$ as highlighted in Fig. 8 and let $L_{C W}=$ $1.4 \mathrm{~m}$ (having the lowest maximum field and less adverse affect on $\sigma_{E}$ ). The median magnetic field required to achieve the constant emittance of $\epsilon_{x}^{c}=0.780 \mathrm{~nm} \mathrm{rad}$ from the data in Fig. 7 is $1.06 \mathrm{~T}$ with a minimum and maximum of 0.57 and $1.26 \mathrm{~T}$ respectively. The calculated magnetic field for this $\mathrm{CW}$ can then be considered as an ID in the calculation of $\epsilon_{x}$ and $\sigma_{E}$ of Eqs. (3) and (4). The resulting emittance and energy spread is shown in Fig. 9. The flat line of emittance here is tautologic, but nevertheless a necessary check. The penalty in energy spread is a slight shift to higher energy spread and amplification of variation as compared to Fig. 7.

Similarly it is possible to choose an energy spread $\sigma_{E}^{c}$ which is achievable by this method. Choosing $\sigma_{E}^{c} / E=$ 0.08262 will result in the horizontal emittance that varies between 0.771 and $0.783 \mathrm{~nm}$ rad. Here the CW100 field varies between 1.84 and $1.29 \mathrm{~T}$. The resultant emittance is 

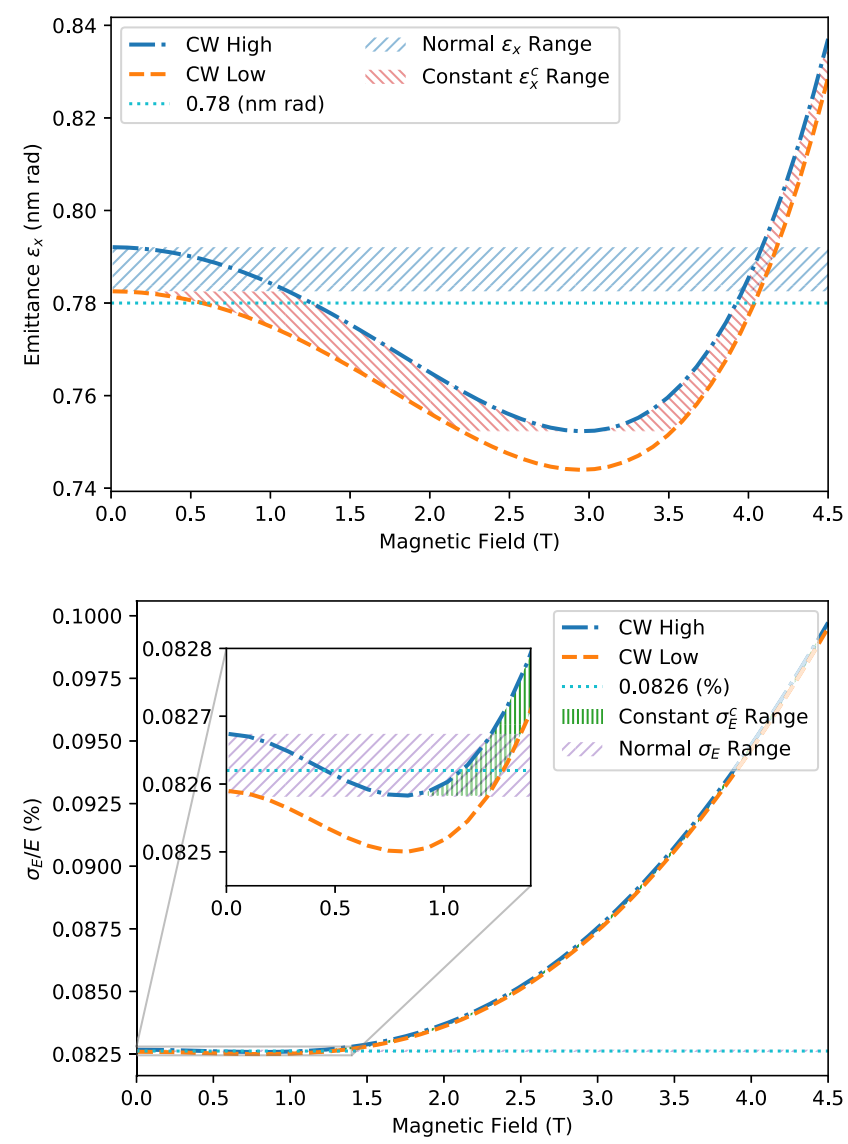

FIG. 10. Total power for all IDs excluding DW100s and including CW100 for constant emittance $\epsilon_{x}^{c}=0.780 \mathrm{~nm} \mathrm{rad}$ and constant energy spread $\sigma_{E}^{c} / E=0.08262$.

slightly lower with nearly the same variation. A comparison of the total ID power output including CW100 is shown for these two scenarios in Fig. 10.

In order to understand the compensation range and consequence of a particular choice of $L_{C W}$ and $\lambda_{C W}$ it is instructive to consider, for example in the case of a desired constant emittance, the minimum and maximum operational emittance configurations. For the NSLS-II data shown herein this is illustrated in the top plot of Fig. 11 as the upper and lower bounds on the Normal $\epsilon_{x}$ Range. The points on the left at zero magnetic field are equivalent to not having a CW. The two lines $C W$ High and $C W$ Low show the emittance for a given $B_{C W}$ starting at the highest and lowest emittance ID configurations. All other ID configurations lie between these two curves.

In order to achieve constant emittance one must be able to compensate both the lowest and highest emittance configurations to the desired compensated emittance. The lowest achievable $\epsilon_{x}^{c}$ occurs at the minimum of $C W$ High curve in Fig. 11. There are then possible operational ranges at higher (around and above $3.5 \mathrm{~T}$ ) and lower field (near $1 \mathrm{~T}$ ) values. The higher range has thus far been typically less desirable due to the negative impact on

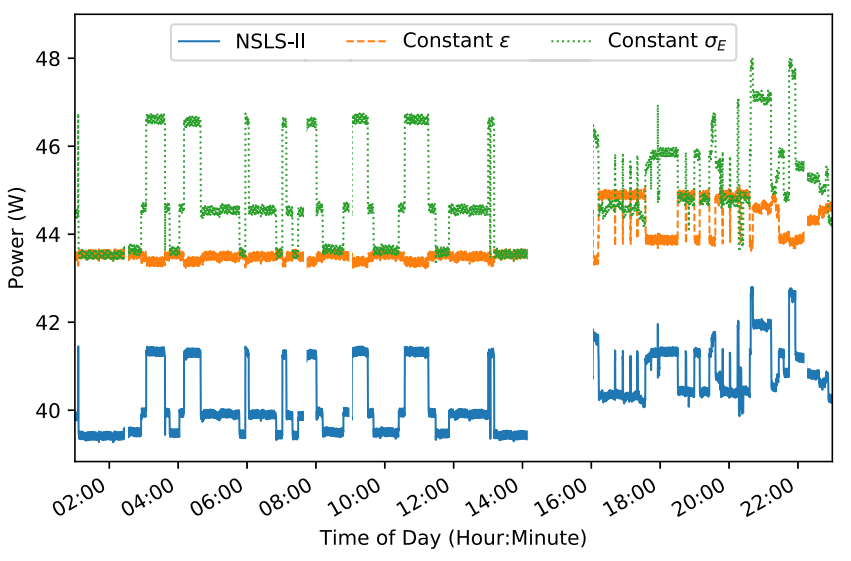

FIG. 11. A $1.4 \mathrm{~m}$ compensation wiggler with a $100 \mathrm{~mm}$ period can be used to compensate the emittance fluctuations from insertion device movement during operations of NSLS-II. The top plot shows the normal emittance range, minimum, maximum, and the emittance compensation capabilities of the hypothetical CW100. The constant $\epsilon_{x}^{c}$ range highlights the range in which a constant compensated emittance is possible. The bottom plot similarly shows the range of $\sigma_{E}$ in operations and the range of possible constant $\sigma_{E}^{c}$.

energy spread, but does have one practical advantage for low emittance rings discussed in Sec. VII. In the lower field range the maximum $\epsilon_{x}^{c}$ is bounded by the minimum uncompensated operational emittance.

\section{COMPENSATION FOR FUTURE LOW EMITTANCE SYNCHROTRONS}

A similar analysis can be performed assuming any lattice. Potential lattice upgrade parameters from Table I are used along with the same time series gap data from NSLS-II shown previously. One can again assess the field requirements and effect on energy spread for different period and length devices shown in Fig. 12. It is evident

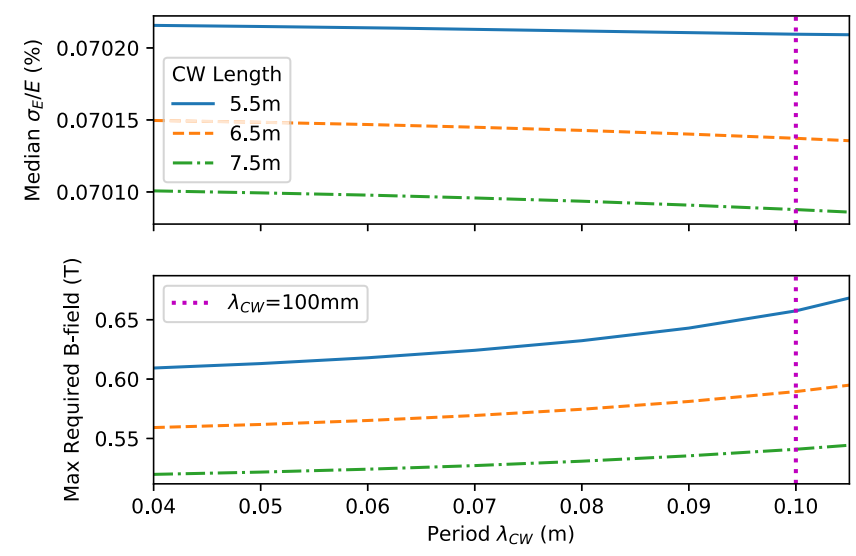

FIG. 12. Effect on median energy spread (top) and maximum magnetic field required (bottom) for constant emittance compensation for different period and length devices for the upgrade lattice parameters in Table I. 


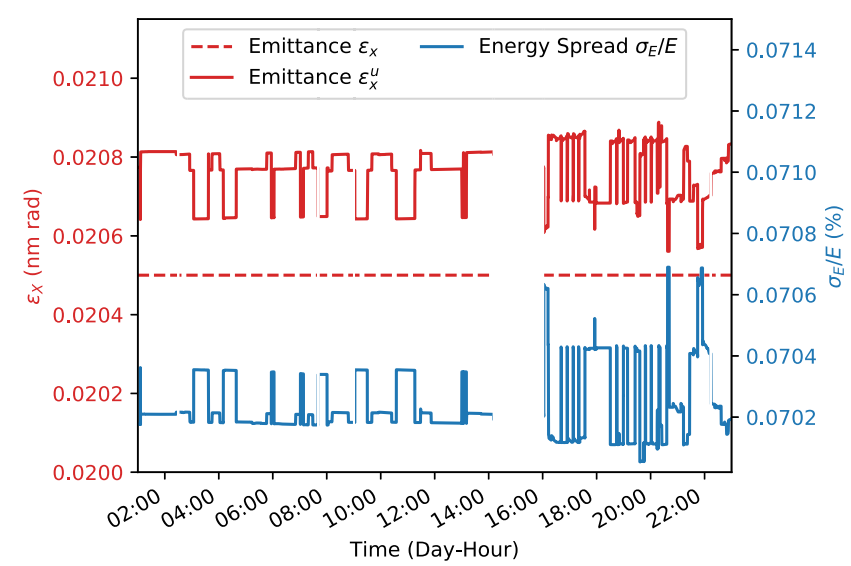

FIG. 13. A compensation wiggler with period $\lambda_{C W}=100 \mathrm{~mm}$ and length $L_{C W}=5.5 \mathrm{~m}$ can be used for either constant emittance compensation (top) or constant energy spread compensation (bottom) for the UL parameters of Table I. The possible ranges for constant emittance or constant energy spread are indicated by the hatched regions while the otherwise normal operational ranges given ID motion are indicated by the shaded regions. Of particular note are the lower field values at which this compensation is possible as compared with a similar device for NSLS-II.

from this that a longer device is again preferred, however the median energy spread is not so dissimilar for the range of lengths shown and the field requirements for the suite of them is realizable. For practical reasons the shortest device is investigated here. Note that the lower bound on length for a period of $100 \mathrm{~mm}$ is not far below $5.5 \mathrm{~m}$ as can be gleaned from Fig. 13 where the $C W$ High curve must have a minimum at or below the minimum of the normal operational range in order to compensate in the lower magnetic field range (and consequently lower emittance range). As the length of the device decreases, its influence diminishes and the minimum of the $C W$ High curve will not penetrate the normal operational range minimum, excluding the low field range as a possibility.

A $5.5 \mathrm{~m} \mathrm{CW} 100$ is capable of achieving either constant emittance compensation or constant energy spread compensation at relatively low magnetic field values. As an example consider $\epsilon_{x}^{c}=0.0205 \mathrm{~nm} \mathrm{rad}$ or $\sigma_{E}^{c} / E=0.0705 \%$. Applying the compensating fields in the low field range of Fig. 13 to the time series gap data of NSLS-II using Eqs. (7) and (8) gives the resulting emittance and energy spread for the two scenarios for the possible upgrade lattice in Fig. 14.

To assess the feasibility of a CW100 compensating device a magnetic model was created using the RADIA [13] software. CW100 is modeled in a standard Halbach [14] configuration using $\mathrm{NdFeB}$ magnets with a remnant field $B_{r}=1.27$. The magnet size is $80 \mathrm{~mm}$ by $50 \mathrm{~mm}$ in the transverse dimension and $25 \mathrm{~mm}$ longitudinally. The simulated magnetic field of this device as a function of
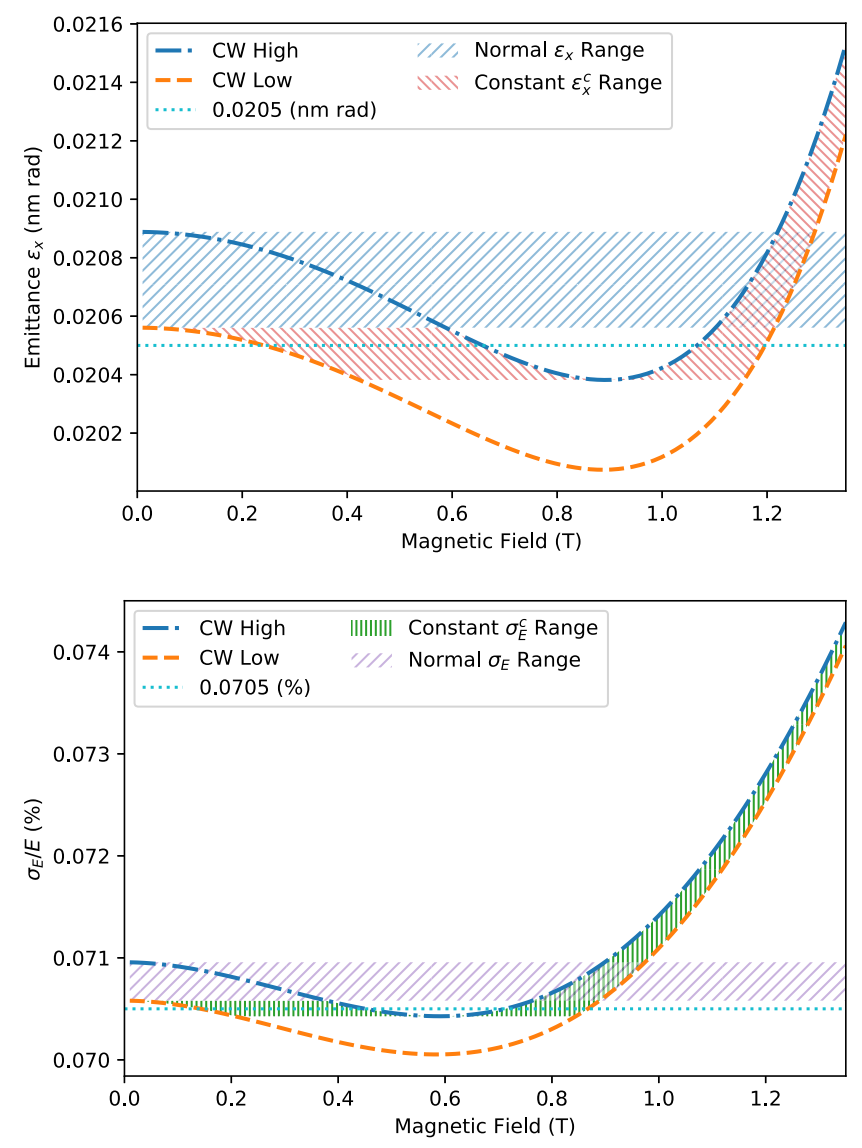

FIG. 14. Emittance $\left(\epsilon_{x}\right)$ and energy spread $\left(\sigma_{E} / E\right)$ for a possible low emittance upgrade lattice (UL) where a $5.5 \mathrm{~m}$ CW100 is providing compensation for constant emittance compensation. The uncompensated emittance is shown as $\epsilon_{x}^{u}$.

gap is given in Fig. 16. For the low field range mentioned the gap range is estimated to be 35.8 to $65.9 \mathrm{~mm}$.

\section{HIGHER FIELD CONSTANT EMITTANCE}

Typically the high field range for constant emittance of Fig. 13 is not used due to a drastic penalty in energy spread which can be seen for instance for NSLS-II in Fig. 11. For a low emittance ring this penalty may not be nearly as severe. Using this high field region for constant emittance compensation results in an energy spread shown in Fig. 15 where it is compared to the low field region energy spread. It is no doubt higher, but not nearly as drastic as would be the case for NSLS-II, and may be tolerable for some applications.

The one advantage of this high field region is the gap range that the compensation occupies shown in Fig. 16. As this is higher field it will have more influence on the synchrotron integrals $I_{2 W .3 W .5 W}$ and lying on the steeper part of the gap versus field range would require smaller physical motion. The gap range (see Fig. 16) for constant emittance is 17.6 to $21.0 \mathrm{~mm}$. The average gap speed for 


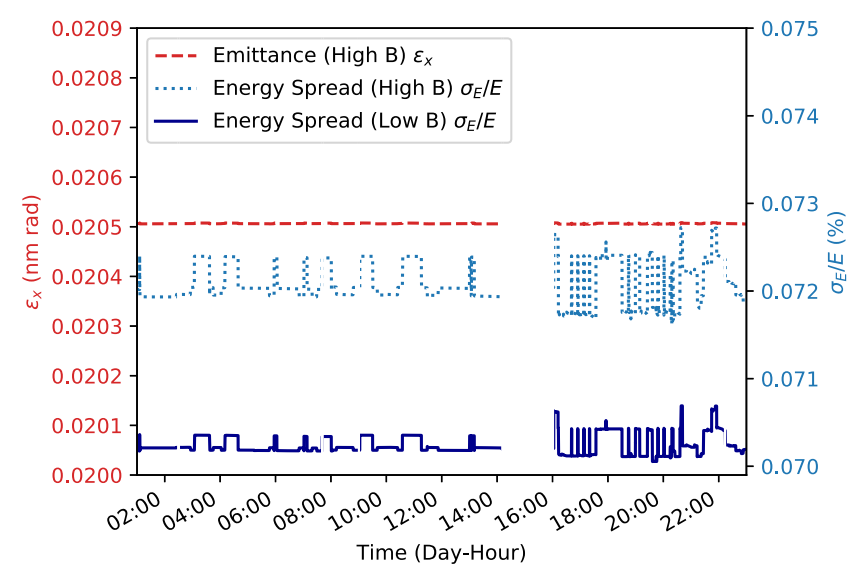

FIG. 15. Using the high field range of Fig. 13 for emittance compensation results in a slightly higher energy spread (High B) as compared to the lower field region (Low B).

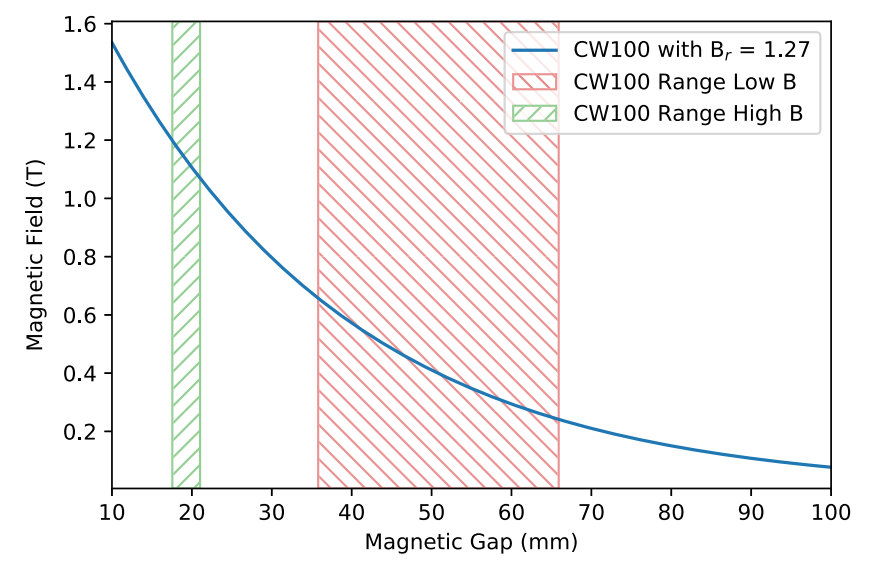

FIG. 16. Magnetic gap ranges for the low magnetic field (Low B) and high magnetic field (High B) ranges for the constant emittance compensation of $\epsilon_{x}^{c}=0.0205 \mathrm{~nm}$ rad of Fig. 13 for a $5.5 \mathrm{~m} \mathrm{CW} 100$.

this compensation based off of the NSLS-II data is $1.3 \mu \mathrm{m} / \mathrm{s}$ with a standard deviation of $20.6 \mu \mathrm{m} / \mathrm{s}$ as compared to $9.2 \mu \mathrm{m} / \mathrm{s}$ with a standard deviation of $146 \mu \mathrm{m} / \mathrm{s}$ for the low field range. The quantile containing $97.7 \%$ of all moves extends to 153 and $1032 \mu \mathrm{m} / \mathrm{s}$ for the high and low range respectively.

Very accurate motion has been achieved for insertion devices [15]. Taking into account acceleration of such large devices, the high field range has some advantage from a mechanical and control system perspective.

\section{CONCLUSION}

Spectral power distributions are computed using the bending magnet and individual insertion device properties to characterize contributions from bending magnets, damping wigglers, gap changing insertion devices, and cumulatively giving a cumulative critical energy equivalent of $\epsilon_{c}=4776 \mathrm{eV}$. Using ID magnetic gap data sampled at $1 \mathrm{~Hz}$ for every insertion device from a 24 hour period of normal operations for NSLS-II coupled with the magnetic field measurements of each ID the radiated power and emittance variation is calculated as a function of time during operations. These data have also been investigated in the context of a $3 \mathrm{GeV}$ low emittance upgrade option. A compensation wiggler is designed and modeled for both which, at different lengths, is capable of achieving a constant emittance or energy spread for either NSLS-II or candidate upgrade low emittance lattice rings. This wiggler conceptual design takes into account the practicalities of current design limitations and from a mechanical and controls perspective these emittance compensating devices are likely feasible.

\section{ACKNOWLEDGMENTS}

This work has been supported by the U.S. Department of Energy, under Contract No. DE-SC0012704.

[1] T. Tanabe, P. Cappadoro, T. Corwin, H. Fernandes, D. A. Harder, Y. Hidaka, C. Kitegi, M. Musardo, and J. Rank, Insertion devices at the national synchrotron light source-ii, Synchrotron Radiat. News 28, 39 (2015).

[2] F. Sannibale, S. Leemann, H. Nishimura, D. Robin, C. Steier, C. Sun, and M. Venturini, Compensation of insertion device induced emittance variations in ultralow emittance storage rings, in 9th International Particle Accelerator Conference, IPAC2018, Vancouver, BC, Canada (JACoW Publishing, 2018).

[3] F. Sannibale, M. Ehrlichman, T. Hellert, S. Leemann, D. Robin, C. Steier, C. Sun, and M. Venturini, Compensation of insertion device induced emittance variations in ultralow emittance storage rings by a dispersion bump in a wiggler, in 10th International Particle Accelerator Conference, IPAC2019, Melbourne, Australia (JACoW Publishing, 2019).

[4] S. Dierker, NSLS-II preliminary design report, Report No. BNL-94744-2007, 2007, https:/www.osti.gov/biblio/ 1010602-nsls-ii-preliminary-design-report.

[5] H. Wiedemann, Particle Accelerator Physics, 3rd ed. (Springer, Berlin Germany, 2007).

[6] M. Borland, ELEGANT: A Flexible SDDS-Compliant Code for Accelerator Simulation (2000), https://www.osti.gov/ biblio/761286.

[7] G. Wang, T. Shaftan, V. Smaluk, Y. Hidaka, O. Chubar, T. Tanabe, J. Choi, S. Sharma, C. Spataro, and N. A. Mesentsev, Complex bend. II. A new optics solution, Phys. Rev. Accel. Beams 22, 110703 (2019).

[8] T. Tanaka and H. Kitamura, SPECTRA: A synchrotron radiation calculation code, J. Synchrotron Radiat. 8, 1221 (2001).

[9] V. Smaluk, Y. Li, Y. Hidaka, T. Tanabe, O. Chubar, L. Wiegart, A. Blednykh, B. Bacha, and T. Shaftan, Effect of undulators on magnet lattice and emittance, Phys. Rev. Accel. Beams 22, 124001 (2019). 
[10] M. Musardo, D. Harder, C. Kitegi, and T. Tanabe, Magnetic measurements of the NSLS-II insertion devices, in 6th International Particle Accelerator Conference, IPAC2015, Richmond, VA, USA (JACoW Publishing, 2015), TUPJE037.

[11] D. Hidas, Computation of synchrotron radiation on arbitrary geometries in 3D with modern GPU, multicore, and grid computing, in Proceedings of the 8th International Particle Accelerator Conference (IPAC 2017), Copenhagen, Denmark, 2017 (JACoW Publishing, Copenhagen, Denmark, 2017), WEPIK121.

[12] W. Cheng, B. Bacha, B. Kosciuk, and D. Padrazo, Jr., Improvements of NSLS-II x-ray diagnostic beam lines, in Proceedings of the 9th International Particle Accelerator Conference (IPAC'18), Vancouver, BC, Canada, 2018, International Particle Accelerator Conference No. 9
(JACoW Publishing, Geneva, Switzerland, 2018), pp. 1837-1840.

[13] O. Chubar, P. Elleaume, and J. Chavanne, A threedimensional magnetostatics computer code for insertion devices, J. Synchrotron Radiat. 5, 481 (1998).

[14] K. Halbach, Physical and Optical properties of Rare Earth Cobalt Magnets (1980), https://www.osti.gov/biblio/ 5068077.

[15] D. Hidas, P. Dean, P. Cappadoro, T. Corwin, J. Escallier, A. Hunt, M. Musardo, J. Rank, C. Rhein, J. Sinsheimer, T. Tanabe, and I. Waluyo, Precision insertion device control and simultaneous monochromator fly scanning for NSLS-II, in Proceedings of the North American Particle Accelerator Conference (NAPAC 2019), Lansing, MI, 2019 (JACoW Publishing, Lansing, MI, USA, 2019), TUPLS09. 